\title{
Apoio Social Como Fator de Proteção para Vitimizações e Desempenho Escolar
}

\author{
Margareth Regina Gomes Veríssimo de Faria ${ }^{1}$, Daniela Sacramento Zanini \\ Pontifícia Universidade Católica de Goiás, Goiânia-GO, Brasil \\ Sonia Regina Pasian \\ Universidade de São Paulo, Ribeirão Preto-SP, Brasil
}

\section{RESUMO}

Este estudo com 426 adolescentes de ensino fundamental I de escolas públicas de Goiânia teve como objetivo avaliar a relação entre apoio social e vitimizações, desempenho escolar e reprovação. Os resultados demonstraram correlações negativas entre sofrer vitimizações e o desempenho em diferentes disciplinas e apoio social e vitimizações. Os meninos apresentaram menor apoio social e mais reprovações que as meninas. Apoio social mostrou-se como fator de proteção para vitimizações e para desempenho escolar. Os resultados apontam para maior vulnerabilidade dos alunos do sexo masculino, pois são os que mais reprovam e possuem menos apoio social percebido.

Palavras-chave: adolescentes; rendimento escolar; família; ensino fundamental.

\section{ABSTRACT - Social Support as a Protective Factor for Victimization and School Performance}

This study with 426 adolescents of Elementary Education I from public schools in Goiânia (Brazil) aimed to assess the relationship between social support and victimization, school performance and reproval. The results showed negative correlations between victimization and performance in different disciplines and social support and victimization. The boys presented less social support and more disapproval than the girls. Social support proved to be a protective factor for victimization and for school performance. The results indicate the vulnerability of male students, since they are the ones that are reproved more and have less perceived social support.

Keywords: adolescents; school performance; family; elementary education.

\section{RESUMEN - Apoyo Social como Factor de Protección para la Victimización y el Rendimiento Escolar}

Este estudio fue realizado con 426 adolescentes pertenecientes a la educación primaria en escuelas públicas de Goiânia (Brasil), el principal objetivo fue el de evaluar la relación entre apoyo social con victimizaciones, rendimiento escolar y repetición de curso. Los resultados demostraron correlaciones negativas entre sufrir victimizaciones y el rendimiento en diferentes disciplinas y apoyo social. Los niños presentaron menor apoyo social y más suspensiones que las niñas. El apoyo social se mostró como factor de protección para victimizaciones y para el rendimiento escolar. Sin embargo, los resultados apuntan a la vulnerabilidad de los alumnos del sexo masculino, pues son los que más repiten curso y poseen menos apoyo social percibido.

Palabras clave: adolescentes; rendimiento escolar; la familia; educación primaria.

A violência contra adolescentes tem sido preocupação mundial demonstrada pelos dados da Organização Mundial da Saúde (OMS), especialmente nos casos de homicídios ocorridos entre adolescentes dos 10 aos 19 anos. $\mathrm{Na}$ comparação internacional com 99 países, o Brasil encontra-se na quarta posição, comprovando a gravidade da situação neste país (Waiselfisz, 2012).

Apesar de estudos apresentarem as consequências da violência, os relatórios sobre violência contra adolescentes demonstram que o número de casos ocorridos contra adolescentes vem aumentando e, paralelamente, as estatísticas do Ministério da Saúde (Brasil, 2010) alertam sobre suas consequências para a saúde de crianças e adolescentes. Segundo a Secretaria de Atenção à Saúde e Secretaria de Vigilância em Saúde, a violência pode gerar problemas sociais, emocionais, psicológicos e cognitivos na adolescência, além contribuírem para o uso e abuso de substâncias como álcool e drogas. Provocam também danos na vida adulta, como problemas de saúde mental e física, dentre outros (Waiselfisz, 2012). Para além da violência convencional, Mayer e Koller (2012) afirmam que fatores relacionados à pobreza econômica ou afetiva

Endereço para correspondência: Rua JG, 07, Quadra 08, Residencial Acácia, S/N, Casa 24 A, Jardim Gardênia, 74394-800, Goiânia,GO.

E-mail: margarethverissimo@gmail.com

Este artigo é um resultado de estudos vinculados ao Projeto PROCAD CAPES: Identidade e vulnerabilidade humana em contextos da Região Centro-Oeste do Brasil, com apoio da CAPES. 
podem impactar negativamente no desenvolvimento de crianças e adolescentes. Whisenhunt, Chibbaro e Perjessy (2014) também afirmam que, mesmo separadamente, a pobreza econômica, ausência de afetividade ou exposição à violência familiar ou comunitária podem impactar negativamente na maturação intelectual, emocional e social de crianças e adolescentes.

A partir de estatísticas sobre violência contra adolescentes brasileiros (Brasil, 2010; Fundo das Nações Unidas para a Infância [UNICEF], 2011, Waiselfisz, 2012), Faria (2015) considerou importante descrever os tipos de violência mais vivenciados pelos adolescentes brasileiros e os prejuízos causados à sua saúde mental. Os resultados desses estudos, em conjunto, demonstraram que a existência de experiências de violência contribui para a ocorrência de outras vitimizações, sendo que sua repetição cria maior impacto negativo na saúde mental dos adolescentes (Faria, 2015; Faria \& Zanini, 2015). Esse tema foi objeto de estudos internacionais (Finkelhor, Hamby, Ormrod, \& Turner, 2005; Finkelhor, Ormrod, \& Turner, 2007) demonstrando que em um único evento podem acontecer várias vitimizações, assim como uma vitimização pode aumentar a probabilidade da ocorrência de outras ocorrências de violência.

As consequências das vitimizações e sua repetição (revitimizações) na saúde mental de adolescentes têm ocupado pesquisadores e organismos governamentais no Brasil há longa data (Brasil, 2010). Autores como Zanini, Forns, Kirchner e Pont (2013) também demonstram preocupação com o tema e afirmam que problemas externalizantes de comportamento estão mais presentes em eventos crônicos do que em eventos agudos de vitimização. Nesse caso, se enquadram as revitimizações ao longo da vida.

Os achados empíricos de Faria (2015) ilustram que situações crônicas de vitimizações podem agravar tanto os sintomas internalizantes, mas principalmente os problemas externalizantes de comportamento. Esses resultados caminham na mesma direção dos encontrados em investigações realizadas fora do Brasil (Cova, Maganto, \& Melipillán, 2005), onde se verificou que os problemas internalizantes e externalizantes de comportamento estão presentes entre adolescentes em situações de adversidades familiares. Há tendência de piora das dificuldades comportamentais ao longo da vida para todos os tipos de vitimizações. Outro dado consistente na literatura científica é o de que sintomas externalizantes também aparecem com maior intensidade na vitimização sexual e naquela violência testemunhada ao longo da vida.

Apesar de a literatura científica constatar os danos provocados pela violência, observa-se que alguns adolescentes permanecem saudáveis, apesar das adversidades. Esse processo é conhecido como resiliência. A resiliência é a capacidade que uma pessoa tem de superar adversidades e manter-se saudável, enquanto outras adoecem nesse mesmo contexto (Rutter, 1985). Dessa forma, é possível afirmar que aquelas variáveis que mediarem e/ou moderarem o risco e as psicopatologias em adolescentes vitimizados serão consideradas variáveis favorecedoras de processos de resiliência. Costa e Bigras (2007) afirmam que crianças e adolescentes podem desenvolver algumas competências que servirão de preditores para o processo de resiliência. Essas competências (capacidade de resolução de problemas; competência social para comunicar-se e demonstrar empatia e resposta positiva; projeto de vida, proposta de futuro, por exemplo) podem ser melhoradas a partir de programas de proteção e promoção de qualidade de vida (Costa \& Bigras, 2007).

Nesse contexto temático faz-se relevante ilustrar investigações que buscaram desenvolver uma rede de apoio social como fator de proteção ao desenvolvimento de adolescentes em contextos de vulnerabilidade social. Exemplo desse tipo de estudo foi desenvolvido na região Centro-Oeste do Brasil por Farias (2016). Seus resultados apontaram que os adolescentes relataram conflitos em relação aos projetos de vida e ao contexto de vulnerabilidade. O contato próximo com o tráfico de drogas foi descrito como fator de risco ao desenvolvimento, enquanto atuaram como fatores de proteção os vínculos saudáveis com familiares e profissionais, a oferta de espaço com atividades culturais, esportivas e lúdicas pelas instituições da comunidade que residiam e frequentavam. As instituições e os profissionais, principalmente aqueles da área de Educação e de Assistência Social, também foram descritos pelos adolescentes como exercendo uma função de apoio na medida em que identificavam e apoiavam necessidades emocionais, psicológicas e materiais destes adolescentes (Farias, 2016).

No contexto do interior do Estado de São Paulo e com objetivo de examinar preditores do desempenho escolar, Fernandes, Leme, Elias e Soares (2018) avaliaram 311 alunos de ensino fundamental I, tendo idade média de 14,0 $\pm 1,2$ anos. Os resultados demonstraram que estudantes do sexo masculino reprovaram mais do que as meninas, mas sem diferenças estatisticamente significativas em função do sexo. Identificaram, como seria esperado, relevante correlação negativa entre a reprovação e o desempenho escolar dos alunos $(r=-0,41 ; p=0,001)$. Além disso, o desempenho escolar apresentou correlações positivas fracas e moderadas com habilidades sociais $(r=0,26 ; p=0,001)$, percepção do apoio social familiar $(r=0,18 ; p=0,02)$ e dos professores $(r=0,22 ; p=0,001)$. Para análise de predição do desempenho escolar dos alunos, foi realizada análise de regressão logística das variáveis do estudo. Evidenciaram que o apoio social do professor e as habilidades sociais dos estudantes predizem o melhor desempenho dos alunos, enquanto a reprovação associa-se negativamente com o rendimento escolar. Os autores destacaram a importância do apoio social dos amigos, familiares e professores no desempenho escolar dos alunos. Ressaltaram que o apoio social dos professores ofereceu resultados estatisticamente significativos para o 
desempenho escolar, mas que todo tipo de apoio social deve ser incentivado no contexto das escolas (Fernandes et al., 2018).

Ao examinar o contexto do ensino fundamental II na região Nordeste do Brasil, mais especificamente no Ceará, Costa, Zeitoune, Queiroz, García e García (2015) focalizaram a análise das percepções de adolescentes sobre as redes de apoio a suas necessidades de saúde. Recorreram à estratégia de grupos focais (três grupos) desenvolvidos com 36 adolescentes cearenses. Os resultados mostraram que a família nuclear foi considerada como uma das fontes de apoio da maior parte dos adolescentes. Quando a família não conseguia dá-lo, ele era buscado em redes secundárias, constituídas pelas relações formais com diferentes serviços, incluindo os serviços de saúde disponíveis aos estudantes.

Essa hipótese da influência positiva do apoio social familiar no contexto do desempenho escolar foi examinada no Brasil por Costa, Montiel, Bartholomeu, Murgo e Campos (2016). Avaliaram 102 crianças do ensino fundamental numa escola do interior do Estado do Maranhão, numa região desfavorecida socioculturalmente no país, utilizando, entre outros recursos, o Inventário de Percepção de Suporte Familiar. Concluíram que quanto mais a criança percebe o ambiente familiar como autônomo, confiável, privativo e livre nas relações entre seus membros, melhor seu desempenho nas disciplinas de português na escola. A percepção que a criança referia sobre sua família pareceu favorecer autonomia, confiança e liberdade nas relações interpessoais. Os autores interpretaram os achados na direção de que o apoio dos pais tende a estimular autonomia e expressão de sentimentos nas crianças, gerando clima de segurança que favorece o bom desempenho escolar (Costa et al., 2016).

Depreende-se, dessa forma, que o apoio social é variável considerada relevante em diferentes contextos, não apenas no Brasil. Contar com rede de apoio e ter a iniciativa de enfrentar diretamente os problemas cotidianos aumentam as probabilidades de resiliência diante de eventos de vida em geral e diante de um problema específico (Manciaux, Vanistendael, Lecomte, \& Cyrulnik, 2003; Olson, Bond, Burns, Wella-Brodrick, \& Sawyer, 2003; Rutter, 2006). Além do apoio social, variáveis como coping e algumas características pessoais, como a autoeficácia, fazem parte desse processo positivo de enfrentamento das adversidades. Há evidências de que ter uma rede de apoio social favorece que o indivíduo enfrente diretamente seus problemas, o que, por sua vez, facilita maior sensação de bem-estar psicológico (Schwarzer \& Knoll, 2007), retroalimentando esse processo.

O apoio social é apontado como variável protetora e favorecedora de preservação da saúde, promovendo na pessoa sentimentos de pertencimento ao grupo social, contribuindo para os processos de adaptação ao contexto de vida (Cia, Pamplin, \& Williams, 2008). Além disso, o apoio social contribui para a pessoa se sentir mais capaz de enfrentar seus problemas, buscando estratégias mais diretas de resolução dos desafios cotidianos, reforçando, por fim, sua acomodação à realidade disponível (Schwarzer \& Leppin, 1991).

Apesar da importância do suporte social no desenvolvimento humano, a faixa etária adolescente, com suas múltiplas vivências de mudanças biopsicossociais, tem sido menos estudada no Brasil numa perspectiva de relações entre variáveis ambientais de violência e rendimento escolar. Diante dessa realidade e da relevância social do tema, este estudo tem como objetivo descrever e interpretar relações entre apoio social e os contextos de vitimizações, reprovação e desempenho acadêmico de adolescentes de ensino fundamental de escolas públicas da região Centro-Oeste do Brasil, especificamente em Goiânia (GO).

\section{Método}

Este estudo é recorte de um projeto de pesquisa mais amplo aprovado no Comitê de Ética em Pesquisa da Pontifícia Universidade Católica de Goiás (CAAE n ${ }^{\circ}$ : 0153.0.168.000-06). Os cuidados técnicos e éticos inerentes à investigação científica foram devidamente realizados ao longo desse trabalho.

\section{Participantes}

Participaram do estudo 426 adolescentes estudantes do ensino fundamental I, após autorização dos seus responsáveis e o seu próprio assentimento com a pesquisa. Eram estudantes de quatro escolas públicas de diferentes regiões da cidade de Goiânia (GO), selecionadas por viabilidade prática, constituindo amostra de conveniência. Os participantes tinham idade média de 13,9 $\pm 1,4$ anos, sendo 232 meninas (54,5\%) e 194 meninos (45,5\%). Do total de participantes $(n=426), 27 \%$ haviam vivido alguma reprovação escolar, com diferença estatisticamente significativa em função do sexo. Entre os meninos o índice de reprovação atingiu $41,8 \%$, enquanto entre as meninas ocorreu em 14,7\% $(t=-6,570 ; p \leq 0,001)$.

\section{Instrumentos}

Questionário de Vitimização Juvenil (JVQ) (Finkelhor et al., 2005), versão traduzida por Faria e Zanini (2011). A versão original contém 34 itens divididos em cinco crivos. Na versão traduzida para o português, foram acrescentados cinco itens sobre vitimização virtual. No total, o Questionário de Vitimização Juvenil (versão brasileira) contém 39 itens e avalia seis tipos de vitimização (Vitimização por Crime Convencional, Vitimização por Maus Tratos, Vitimização por Pares, Vitimização Sexual, Vitimização Indireta/Testemunhada e Vitimização Virtual). As respostas eram dadas em "sim" ou "não" para vitimização no "último ano" (pontual) e "ao longo da vida" (crônica). 
Escala de Apoio Social. Originalmente construída para o Medical Outcomes Study (MOS) (Sherbourne \& Stewart, 1991), adaptada para o português por Griep, Chor, Faerstein, Werneck e Lopes (2005). A escala de 19 itens, em formato Likert, varia de 1 (nunca) a 5 (sempre), cobrindo quatro dimensões, segundo análise fatorial: 1. apoio material; 2 . apoio afetivo; 3 . apoio emocional e de informação; 4. apoio interação social positiva.

Roteiro sobre dados sociodemográficos (idade, sexo) e perguntas sobre o desempenho em disciplinas escolares.

\section{Procedimentos}

Os estudantes foram convidados a participarem do estudo após explicação pela primeira pesquisadora, em sala de aula, dos objetivos do estudo, participação voluntária, anonimato das respostas e no tratamento dos dados. Após envio do Termo de Consentimento Livre e Esclarecido a seus responsáveis, aqueles estudantes que concordaram em participar da pesquisa leram e assinaram o Termo de Assentimento ao estudo. Foram programadas sessões coletivas de aplicação dos instrumentos, respeitando os grupos de sala de aula e procedimentos escolares. Os instrumentos foram administrados nos horários de aula nas instituições de ensino, por psicólogos e estudantes de Psicologia previamente treinados, conforme suas diretrizes técnicas específicas.
A coleta de dados foi realizada em dois momentos devido à extensão dos instrumentos. Ao se realizar coleta de dados em dois momentos, corre-se o risco de perda amostral. Assim, foi possível colher o roteiro de dados sociodemográficos, desempenho escolar e JVQ de 426 voluntários. No entanto, na aplicação da Escala de Apoio Social, foram coletados 323 casos. O total de participantes que responderam ao conjunto de instrumentos foi de 320 adolescentes.

Os resultados foram sistematizados de acordo com sua padronização técnico-científica, digitados em planilha computacional (Microsoft Excel) para posteriores análises estatísticas. Recorreu-se, para tanto, ao software IBM SPSS Statistics, adotando-se nível de significância de 0,05 nas análises estatísticas.

\section{Resultados}

Inicialmente têm-se os resultados descritivos relativos à vitimização dos 426 adolescentes avaliados, conforme informações do JVQ brasileiro, distribuídos em função do sexo (232 meninas e 194 meninos). Na Tabela 1 são comparados somente os participantes que sofreram vitimizações (168 casos no último ano e 169 casos ao longo da vida), eliminando-se aqueles que não foram vitimizados para essa análise.

Tabela 1

Vitimização entre Adolescentes em Função do Sexo

\begin{tabular}{|c|c|c|c|c|c|c|c|c|}
\hline \multirow{2}{*}{ Vitimização } & \multirow{2}{*}{ Sexo } & \multicolumn{2}{|c|}{$\mathrm{n}^{\circ}$ casos } & \multicolumn{2}{|c|}{ Eventos } & \multicolumn{3}{|c|}{ Comparação estatística } \\
\hline & & $f$ & $\%$ & Média & DP & $t$ & $p$ & $g \mathrm{gl}$ \\
\hline \multirow{2}{*}{ Último ano } & Fem. & 105 & 45,3 & 7,9 & 5,8 & \multirow{2}{*}{0,676} & \multirow{2}{*}{0,500} & \multirow{2}{*}{166} \\
\hline & Masc. & 63 & 32,5 & 7,3 & 5,6 & & & \\
\hline \multirow{2}{*}{ Ao longo da vida } & Fem. & 105 & 45,3 & 9,5 & 6,4 & \multirow{2}{*}{$-0,204$} & \multirow{2}{*}{0,839} & \multirow{2}{*}{167} \\
\hline & Masc. & 64 & 33,0 & 9,7 & 6,9 & & & \\
\hline
\end{tabular}

Embora não seja o foco central deste trabalho, cabe destacar que o tipo mais frequente de vitimização entre os adolescentes foi Vitimização Indireta/Testemunhada no Último Ano (80,8\%). Já Crime Convencional e Vitimização Indireta/Testemunhada predominaram ao Longo da Vida (85,0\%).

Apesar da incidência de vitimização ser maior entre estudantes do sexo feminino, atingindo quase metade das meninas avaliadas, não houve diferença estatisticamente significativa em relação ao sexo masculino. Nota-se que o relato sobre o último ano é muito parecido com as vivências dos estudantes ao longo da vida em termos de incidência dessa experiência de vitimização entre os adolescentes.
No tocante à quantidade de experiências de violência vividas pelos adolescentes, dentre aqueles que referiram vitimização, nota-se inexistência de diferenças estatisticamente significativas entre meninos e meninas. É interessante notar que, entre os vitimizados, a incidência é alta, atingindo média de 7-8 ocorrências no último ano e 9-10 episódios ao longo da vida, independentemente do sexo. Em outras palavras, caracterizam-se como adolescentes polivitimizados.

Visto que o sexo não pareceu influenciar os achados referentes à experiência de vitimização entre os estudantes, procurou-se examinar a distribuição dos casos em função do histórico acadêmico de reprovação. A Tabela 2 ilustra esses achados. 
Tabela 2

Vitimização entre Adolescentes em Função do Desempenho Acadêmico

\begin{tabular}{|c|c|c|c|c|c|c|c|c|}
\hline \multirow{2}{*}{ Vitimização } & \multirow{2}{*}{ Reprovação } & \multicolumn{2}{|c|}{$\mathrm{n}^{\circ}$ casos } & \multicolumn{2}{|c|}{ Eventos } & \multicolumn{3}{|c|}{ Comparação estatística } \\
\hline & & $f$ & $\%$ & Média & $D P$ & $t$ & $p$ & $g l$ \\
\hline \multirow{2}{*}{ Último ano } & Sim & 33 & 19,8 & 8,8 & 5,9 & \multirow{2}{*}{$-1,338$} & \multirow{2}{*}{0,183} & \multirow{2}{*}{165} \\
\hline & Não & 134 & 80,2 & 7,3 & 5,6 & & & \\
\hline \multirow{2}{*}{ Ao longo da vida } & Sim & 33 & 19,6 & 10,8 & 7,7 & \multirow{2}{*}{$-1,246$} & \multirow{2}{*}{0,214} & \multirow{2}{*}{166} \\
\hline & Não & 135 & 80,4 & 9,2 & 6,3 & & & \\
\hline
\end{tabular}

Nota-se que a experiência mais recente de vitimização (último ano) ou ao longo da vida não diferencia os estudantes em termos do índice de reprovação. Naqueles casos onde houve reprovação acadêmica, houve índice de aproximadamente $20,0 \%$ de estudantes vitimizados, atualmente ou durante sua história de vida. Também cabe destacar que, apesar de inexistirem diferenças estatisticamente significativas entre esses estudantes vimitizados, os que mais reprovam são os que possuem maiores médias de vitimização no último ano e ao longo da vida.

Ainda em relação ao desempenho escolar e às vitimizações, análises de correlação indicaram que quanto menos vitimizações sofridas, maior o desempenho escolar. As vitimizações no último ano correlacionaram-se negativamente com rendimento escolar nas disciplinas de português $(r=-0,275, p=0,02, N=71)$, matemática $(r=-0,237, p=0,04, \mathrm{~N}=75)$ e outras não descritas $(r=-0,497, p=0,004, \mathrm{~N}=31)$. Em relação às vitimizações ao longo da vida as correlações também se apresentaram negativas para desempenho nas disciplinas em português $(r=-0,242, p=0,04, N=70)$, matemática $(r=-0,374, p=0,001, N=73)$ e em outras disciplinas $(r=-0,497, p=0,004, N=31)$. Esses resultados indicam que os adolescentes que sofreram menos vitimizações também apresentaram melhores desempenhos nas disciplinas do ensino fundamental I.

A análise relativa aos indicadores de apoio social evidenciou resultados estatisticamente diferentes em função do sexo dos estudantes $(t=4,763 ; p \leq 0,001)$. As meninas referiram indicadores de maior apoio social $(M=4,3$; $D P=0,7)$ do que os meninos $(M=3,9 ; D P=0,9)$.

A média de apoio social dos estudantes que não reprovam $(N=237 ; M=4,2 ; D P=0,8)$ foi maior daqueles com experiência de reprovação escolar $(N=83 ; M=3,8$; $D P=1,0)$. Houve diferença estatística significativa entre esses dois grupos $(t=-3,833 ; p \leq 0,001)$, afirmando a relevância do contexto de apoio social para o bom rendimento na escola.

Há que apontar ainda que a escala geral de apoio social se correlacionou negativamente $(r=-0,191)$ com a vitimização ao longo da vida. Os resultados apontaram que quanto maior o apoio social geral recebido, menor a vitimização ao longo da vida. Realizou-se análise de regressão simples para examinar essas experiências de vida dos adolescentes, utilizando como variável o critério de cronicidade da vitimização (ao longo da vida) e como variável antecedente Apoio Social. Os resultados estão apresentados na Tabela 3 .

Tabela 3

Regressão Simples Relacionando Vitimização e Apoio Social

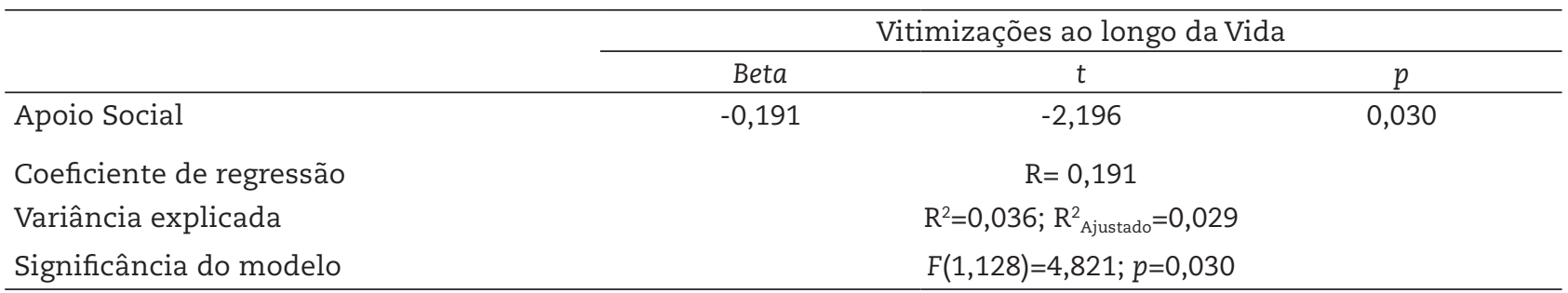

Notou-se que indicadores de reduzido apoio social explica 2,9\% das vitimizações ao longo da vida. Embora o índice de variância explicada possa ser considerado baixo, possui clara relevância social por se tratar de estudantes que se encontram na adolescência, momento relevante para seu pleno desenvolvimento. Os resultados dessa análise de regressão demonstraram a importância do apoio social como fator de proteção para as vitimizações ao longo da vida. Desse modo, tem-se achados empíricos que apontam que a presença de recursos de apoio social tende a favorecer menos vitimizações no curso de seu desenvolvimento. 


\section{Discussão}

Este estudo procurou descrever e interpretar relações entre apoio social e os contextos de vitimizações, reprovação e desempenho acadêmico de adolescentes de ensino fundamental de escolas públicas de Goiânia (GO). Os resultados demonstraram que meninos sinalizaram menos apoio social que meninas e apresentaram mais reprovações. Por outro lado, os adolescentes que sofreram menos vitimizações foram também os que apresentaram melhores resultados nas disciplinas escolares. As vitimizações (no último ano e ao longo da vida) correlacionaram-se negativamente com o bom desempenho nas atividades acadêmicas. $\mathrm{E}$, ainda, quem não possui histórico de reprovação na escola referiu índices de apoio social mais elevados em relação aos estudantes com reprovação acadêmica.

Há vários anos a preocupação com as vivências de violência e suas consequências para os adolescentes são estudadas em inúmeros contextos, inclusive no Brasil. O Ministério da Saúde (Brasil, 2010) apresentou evidências sobre as consequências da violência repetida para a saúde mental dos adolescentes há mais de dez anos. Zanini et al. (2013) também demonstraram associação entre essas variáveis, apontando inclusive para os problemas decorrentes de experiências crônicas de exposição à violência e de vitimização. Os resultados deste estudo, por sua vez, reforçaram a relevância social dessa temática, apontando que o apoio social pode funcionar como fator de proteção para adolescentes vitimizados ao longo da vida. De outro modo, não sofrer vitimizações favorece bom desempenho em disciplinas escolares, como matemática e português, ou seja, adolescentes menos vitimizados apresentam melhor desempenho acadêmico.

Percebe-se que o maior apoio social está negativamente relacionado com vitimizações ao longo da vida e a menos reprovação escolar. As atuais evidências empíricas corroboram achados referidos por Costa et al. (2015) referentes à importância do apoio social para o adequado desenvolvimento infantil. O estudo de Fernandes et al. (2018) também apresentou correlações positivas entre desempenho escolar com habilidades sociais, percepção do apoio social familiar e dos professores.

Apoio social apresentou-se, portanto, como fator de proteção para a vitimização de adolescentes, confirmando o que alguns estudiosos vêm apresentando em seus trabalhos, como o de Costa et al. (2016). Eles verificaram que quanto mais a criança percebe o ambiente familiar como autônomo, confiável, privativo e livre nas relações entre seus membros, melhor é seu desempenho nas disciplinas de português. Os autores ainda valorizam a importância do apoio social dos amigos, familiares e professores no desempenho escolar dos alunos. Há que se ressaltar que todo tipo de apoio social deve ser incentivado para apoiar o desenvolvimento acadêmico, como apontado por Fernandes et al. (2018).

Desse modo, este estudo reforça os resultados da literatura científica sobre a importância do apoio social como fator de proteção para a vida de adolescentes, impactando em seu contexto escolar de forma significativa. Por fim, atenção especial merece ser oferecida aos alunos do sexo masculino, pois apresentaram maiores índices de reprovação, menor apoio social e menor desempenho acadêmico, sugerindo maior vulnerabilidade ao longo da adolescência.

\section{Referências}

Brasil. Ministério da Saúde. Secretaria de Atenção à Saúde. Secretaria de Vigilância em Saúde (2010). Impacto da Violência na Saúde das Crianças e Adolescentes. Brasília (DF): Ministério da Saúde.

Cia, F., Pamplin, R. C. O., \& Williams, L. C. A. (2008). O impacto do envolvimento parental no desempenho acadêmico de crianças escolares. Psicologia em Estudo, 13(2), 351-360. doi: 10.1590/S1413-73722008000200018

Costa, K., Montiel, J. M., Bartholomeu, D., Murgo, C. S., \& Campos, N. R. (2016). Percepção do suporte familiar e desempenho em leitura e escrita de crianças do ensino fundamental. Revista de Psicopedagogia, 33(101), 154-163.

Costa, M. D. C. O., \& Bigras, M. (2007). Mecanismos pessoais e coletivos de proteção e promoção da qualidade de vida para a infância e adolescência. Ciência e Saúde Coletiva, 12(5), 1101-1109. doi: 10.1590/S1413-81232007000500002

Costa, R., Zeitoune, R., Queiroz, M., Gómez García, C., \& Ruiz García, M. (2015). Redes de apoio ao adolescente no contexto do cuidado à saúde: Interface entre saúde, família e educação. Revista da Escola de Enfermagem da USP, 49(5), 741-747. doi: 10.1590/S0080623420150000500005.

Cova, S., Maganto, M., \& Melipillán, A. (2005). Adversidad familiar y desarrollo de trastornos internalizados y externalizados en preadolescentes. Revista chilena de Neuro-psiquiatría, 43(4), 287-296.

Faria, M. R. G. V., \& Zanini, D. S. (2011, Julho). Análise da Compreensão dos Itens do Questionário de Vitimização (JVQ) após Tradução para o Português. Resumo apresentado na 63a Reunião Anual da SBPC, UFG-Goiânia, GO. Recuperado de http://www.sbpcnet.org.br/ livro/63ra/resumos/resumos/6155.htm

Faria, M. R. G. V., \& Zanini, D. S. (2015). Incidences and occurences of future types of victimization in adolescents. Psychology, 6(10), 1249-1254. doi: 10.4236/psych.2015.610122

Faria, M. R. G. V. (2015). Polivitimização e revitimização em adolescentes: Avaliação e consequências para a saúde mental (Tese de doutorado). Pontifícia Universidade Católica de Goiás, Goiás. 
Farias, R. (2016). Desenvolvimento psicossocial de adolescentes em contextos de vulnerabilidade social (Tese de doutorado). Universidade Federal de Santa Catarina, Florianópolis.

Fernandes, L. M., Leme, V. B. R., Elias, L. C. S., \& Soares, A. B. (2018). Preditores do desempenho escolar ao final do ensino fundamental: Histórico de reprovação, habilidades sociais e apoio social. Temas em Psicologia, 26(1), 215-228. doi: 10.9788/TP2018.1-09Pt

Finkelhor, D., Hamby, S. L., Ormrod, R., \& Turner, H. (2005). The juvenile victimization questionnaire: Reliability, validity and national norms. Child Abuse \& Neglect, 29(4), 383-412. doi: 10.1016/j.chiabu.2004.11.001

Finkelhor, D., Ormrod, R. K., \& Turner, H. A., (2007). Re-victimization patterns in a national longitudinal sample of children and youth. Child Abuse \& Neglect, 31(5), 479-502. doi: 10.1016/j.chiabu.2006.03.012

Fundo das Nações Unidas para a Infância (2011). O direito de ser adolescente: Oportunidade para reduzir vulnerabilidades e superar desigualdades. Brasília, DF: UNICEF.

Griep, R., Chor, D., Faerstein, E., Werneck, G., \& Lopes, C. (2005). Validade de construto de Escala de Apoio Social do Medical Outcomes Study adaptada para o português no Estudo Pró-Saúde. Cadernos de Saúde Pública, 21(3), 703-714.

Manciaux, M., Vanistendael, S., Lecomte, J., \& Cyrulnik, B. (2003). La resiliencia: Estado de la cuestión. Em M. Manciaux (Ed.). La resiliencia: resisitir y rehacerse (pp. 17-27). Barcelona: Gedisa.

Mayer, L. R., \& Koller, S. H. (2012). Rede de apoio social e representação mental das relações de apego de crianças e adolescentes vítimas de violência doméstica. Em L. F. Habigzang, \& S. H. Koller (Orgs.), Violência contra crianças e adolescentes: teoria, pesquisa e prática (pp. 21-32). Porto Alegre, RS: Artmed.

Olson, C. A., Bond, L., Burns, J. M., Vella-Brodrick, D. A., \& Sawyer, S. M. (2003). Adolescent resilience: a concept analysis. Journal of Adolescence, 26(1), 1-11. doi: 10.1016/S0140-1971(02)00118-5

Rutter, M. (1985). Resilience in the face of adversity: Protective factors and resistance to psychiatric disorder. British Journal of Psychiatry, 147(6), 598-611. https://doi.org/10.1192/bjp.147.6.598

Rutter, M. (2006). Implications of resilience concepts for scientific understanding. Annals New York Academy of Sciencies, 1094(1), 1-12.

Sherbourne, C. D., \& Stewart, A. L. (1991). The MOS social support survey. Social Science \& Medicine, 32(6), 705-714. doi: 10.1016/02779536(91)90150-B

Schwarzer, R., \& Knoll, N. (2007). Functional roles of social support within the stress and coping process: a theoretical and empirical overview. International Journal of Psychology, 42(4), 243-252. doi: 10.1080/00207590701396641

Schwarzer, R., \& Leppin, A (1991). Social Support and health: A theoretical and empirical overview. Journal of Social and Personal Relationships, 6(99), 99-127. doi: 10.1177/0265407591081005

Waiselfisz, J. J. (2012). Mapa da violência 2012: Crianças e adolescentes do Brasil. 1a Edição. Rio de Janeiro (RJ): FLACSO Brasil.

Whisenhunt, J. L., Chibbaro, J. S., \& Perjesy, C. (2014) Psychosocial factors in adolescent self-injury. Em M. T. Garrett (Org.). Youth and Adversity: Psychology and influences of child and adolescente resilience and coping. New York, NY: Nova Biomedical.

Zanini, D. S., Forns, M., Kirchner, T., \& Pont, E. (2013). Personalidade Ansiosa e Problemas de comportamento: Influência dos eventos da vida e apoio social. Em: L. de F. Carvalho \& R. Primi. (Orgs.), Perspectivas em psicologia dos transtornos da personalidade: Implicações teóricas e práticas (pp. 402-420). São Paulo, SP: Casa do Psicólogo.

\section{Sobre as autoras}

Margareth Regina Gomes Veríssimo de Faria é psicóloga (PUC Goiás), doutora em Psicologia pela Pontifícia Universidade Católica de Goiás (PUC Goiás) e pós-doutora pela USP - Ribeirão Preto. Atualmente, é professora colaboradora no Programa de Pós-Graduação Stricto Sensu em Psicologia da Pontifícia Universidade Católica de Goiás.

Daniela Sacramento Zanini é psicóloga (PUC Goiás), com doutorado em Psicologia Clínica e da Saúde e pós-doutorado pela Universidad de Barcelona (Espanha). Atualmente, é professora adjunto II da Pontifícia Universidade Católica de Goiás na graduação e pósgraduação Stricto Sensu em Psicologia (mestrado e doutorado).

Sonia Regina Pasian é psicóloga pela Universidade de São Paulo (USP), com doutorado em Ciências (Saúde Mental) e livre docência pela USP. Atualmente, é professora titular da Faculdade de Filosofia, Ciências e Letras de Ribeirão Preto (FFCLRP) da Universidade de São Paulo, onde coordena o Centro de Pesquisas em Psicodiagnóstico. 